\title{
Sanger Sequencing
}

National Cancer Institute ( $\mathrm{NCl})$

\section{Source}

National Cancer Institute (NCI). Sanger sequencing.

A low-throughput method used to determine a portion of the nucleotide sequence of an individual's genome. This technique uses polymerase chain reaction (PCR) amplification of genetic regions of interest followed by sequencing of PCR products. 